\title{
Rich Intrinsic Image Decomposition of Outdoor Scenes from Multiple Views
}

\author{
Pierre-Yves Laffont, Adrien Bousseau and George Drettakis
}

\section{ApPendiX: IlLUMinANT CALIBRATION}

In this appendix, we describe the details of the illuminant calibration step for the sky and the sun.

First, because our model separates sun light from sky light, we need to remove sun pixels from the environment map. We define the sun position as the barycenter of the saturated sun pixels, and use inpainting to fill-in these saturated pixels from their neighbors. Since our model also separates sky light from indirect light, we use a standard color selection tool to label sky pixels that will contribute to the sky illumination, while other pixels (building, trees) will contribute to indirect lighting. This is illustrated in Fig. 5c.

Second, we align the environment map and sun with the reconstructed scene. To do so we manually mark a vertical edge of the reconstructed geometry and rotate the environment map and sun until the cast shadow of the virtual edge is aligned with that in the photograph.

Finally, the environment map only captures a scaled version of the scene lighting since the sphere is not perfectly specular. We need to compensate for this scaling factor in each color channel.

In our system the environment map is used to compute both the sky illumination $\mathbf{S}_{\text {sky }}$ and part of the indirect illumination $\mathbf{S}_{\text {ind }}$, the other part being computed from the geometric proxy (see Section 5 for more details). We estimate the color transfer function of the reflective sphere $\mathbf{K}$ (represented as a RGB vector) by taking a photograph of a neutral gray card with known reflectance $\mathbf{R}$ placed in sun shadow. We intentionally place the card at a position where we expect its geometry to be well reconstructed. From the image formation model we have

$$
\begin{aligned}
\mathbf{I} & =\mathbf{R} *\left(\mathbf{S}_{\text {sky }}+\mathbf{S}_{\text {ind }}\right) \\
& =\mathbf{R} *\left(\mathbf{K} * \mathbf{S}_{\text {sky }}^{\text {env }}+\mathbf{K} * \mathbf{S}_{\text {ind }}^{\text {env }}+\mathbf{S}_{\text {ind }}^{\text {proxy }}\right)
\end{aligned}
$$

where $\mathbf{S}^{\text {env }}$ denotes the illumination terms computed from the environment map and $\mathbf{S}^{\text {proxy }}$ the ones computed from the geometric proxy. We use this equation to solve for the unknown $\mathbf{K}$.
We similarly recover the sun radiance $\mathbf{L}_{\text {sun }}$ by taking a second picture of the gray card placed in sunlight $\left(v_{\text {sun }}=1\right)$. From this picture we have

$$
\begin{aligned}
\mathbf{I} & =\mathbf{R} *\left(\mathbf{S}_{\text {sun }}+\mathbf{S}_{\text {sky }}+\mathbf{S}_{\text {ind }}\right) \\
& =\mathbf{R} *\left(v_{\text {sun }} \cos \theta_{\text {sun }} \mathbf{L}_{\text {sun }}+\mathbf{S}_{\text {sky }}+\mathbf{S}_{\text {ind }}\right)
\end{aligned}
$$

where $\mathbf{L}_{\text {sun }}$ is the only unknown.

We could envisage other approaches for this calibration. Cabral et al. [1] use a compass to compute the environment map orientation with respect to the view direction in each photograph. In the work of Yu and Malik [2], a set of photographs of the horizon are taken, and a sky model is fitted. This involves taking several photographs, as opposed to the only two we take of the sphere, and also requires the use of two neutral lens filters to estimate sun radiance. In addition, fitting parameters of sky models can require specific tuning for relighting applications, as was the case for morning and evening sky in the work of Cabral et al. [1].

\section{SUPPLEMENTARY VIDEO}

A supplementary video is attached. It illustrates some applications of our decomposition in image editing software.

\section{SUPPLEMENTARY FIGURES}

In this document we show results on four scenes, for a total of 14 views. For each viewpoint, we provide the input (rectified) image, estimated reflectance, as well as the constraints and estimated total illumination, sun illumination, sky illumination and indirect illumination.

We also show examples of the candidate reflectance curves after clustering, in all of our scenes.

\section{References}

[1] M. Cabral, N. Bonneel, S. Lefebvre, and G. Drettakis, "Relighting photographs of tree canopies," IEEE Transactions on Visualization and Computer Graphics, vol. 17, pp. 1459-1474, 2011.

[2] Y. Yu, P. Debevec, J. Malik, and T. Hawkins, "Inverse global illumination: recovering reflectance models of real scenes from photographs," in SIGGRAPH '99, 1999, pp. 215-224. 

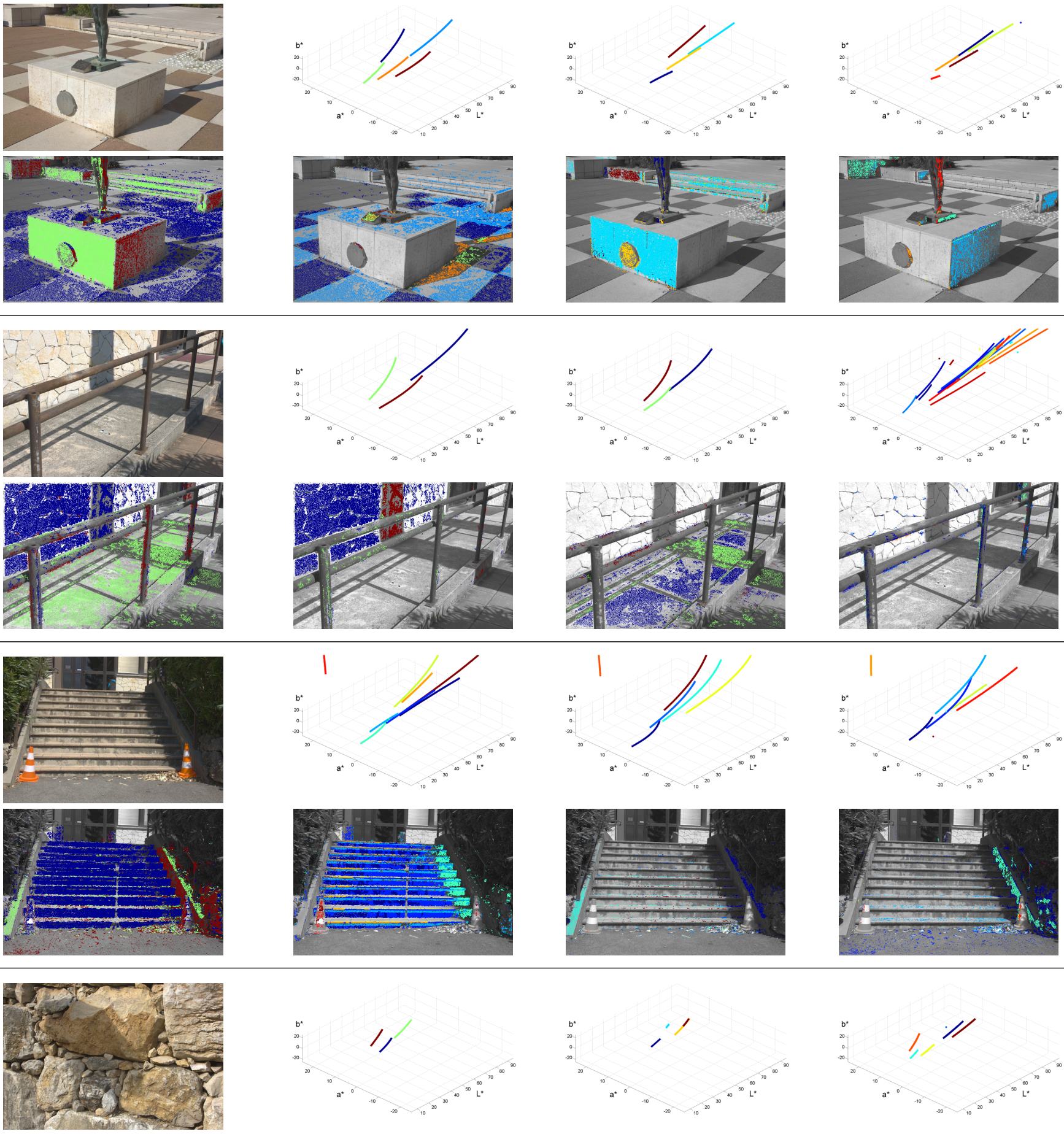

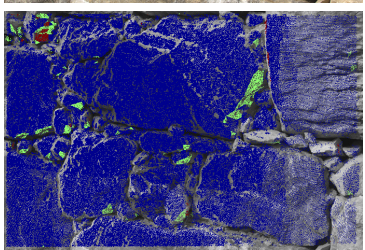

(a) Groups of normal orientation

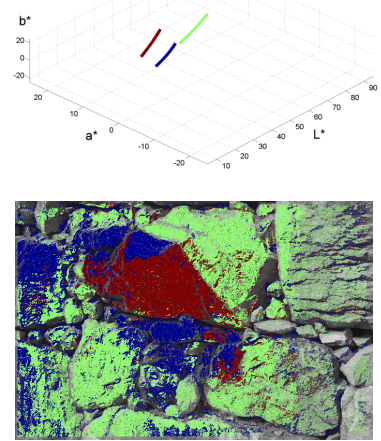

(b) Curve clusters, orientation 1
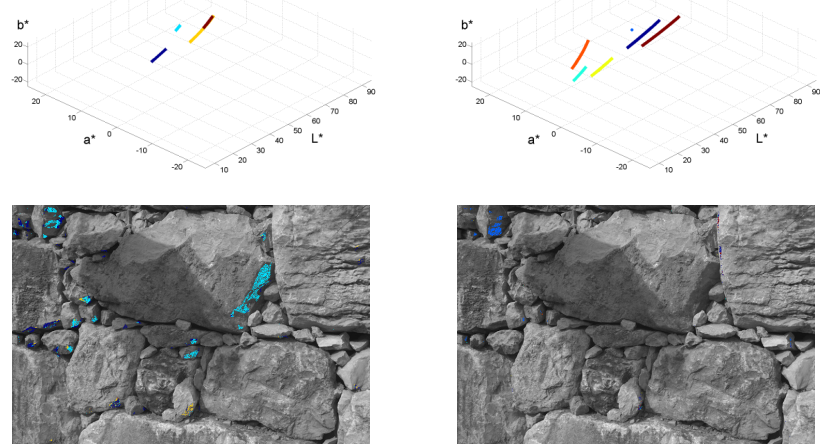

(c) Curve clusters, orientation 2

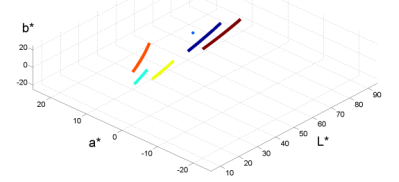

TABLE 1: Clustering and candidate curves. PMVS points are separated into three groups according to the orientation of their normals ( $a$, bottom). Within each group, PMVS points are clustered (b-d, bottom) based on their candidate reflectance curves. Each cluster is then represented by a single representative curve (b-d,top). The clustering parameters were fixed for all scenes. 


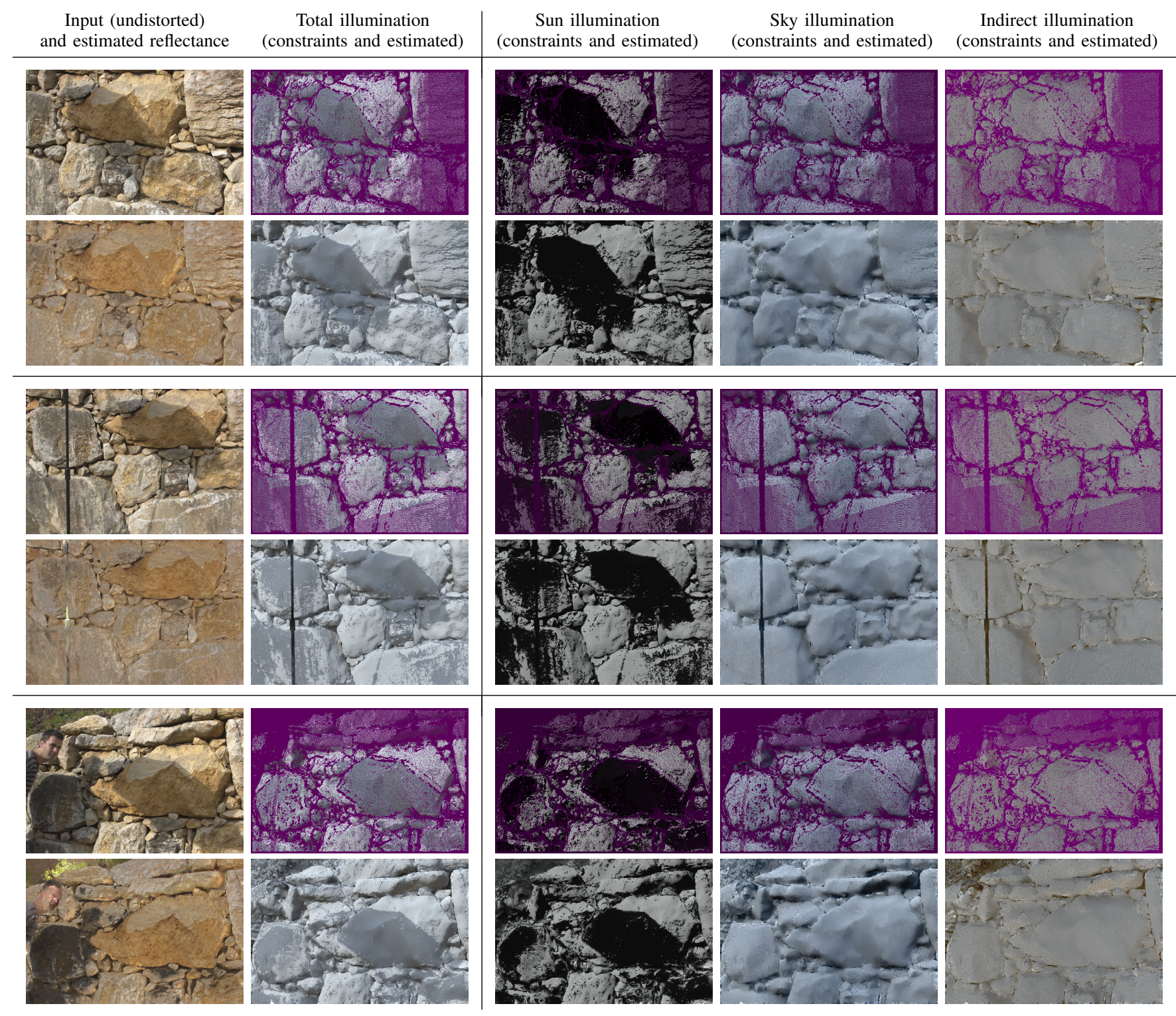

TABLE 2: Results of our decomposition on the Rocks scene. This scene did not require inpainting after the illumination separation. The linear images have been scaled for display, before applying gamma correction with $\gamma=1 / 2.2$; the scaling values were: 0.7 for the input, 1.8 for the reflectance, 0.12 for total illumination and sun illumination, 0.2 for sky illumination, 0.5 for indirect illumination. 


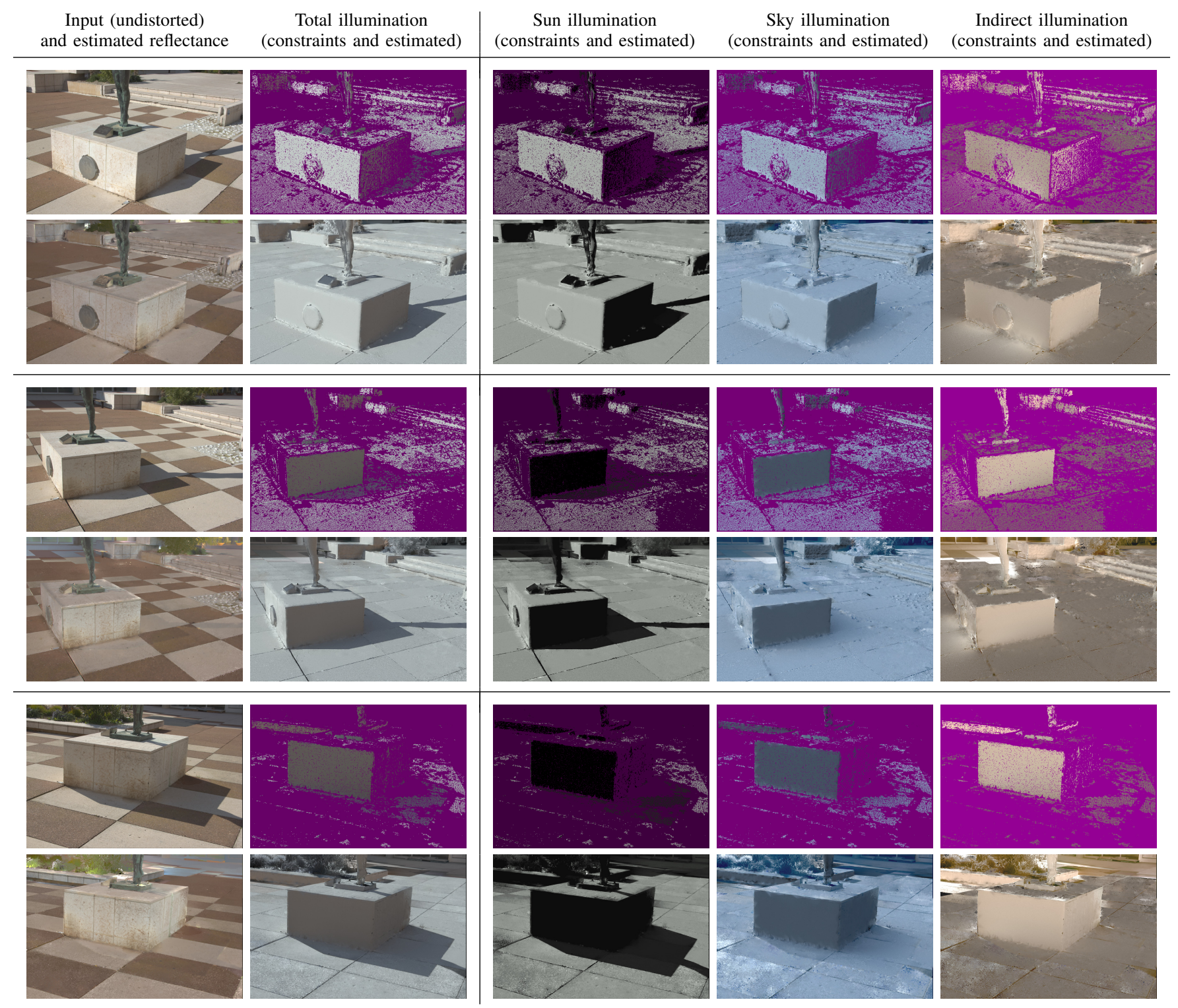

TABLE 3: Results of our decomposition on the Statue scene after final inpainting. The linear images have been scaled for display, before applying gamma correction with $\gamma=1 / 2.2$; the scaling values were: 0.6 for the input, 1 for the reflectance, 0.15 for total illumination and sun illumination, 0.6 for sky illumination, 1 for indirect illumination. 


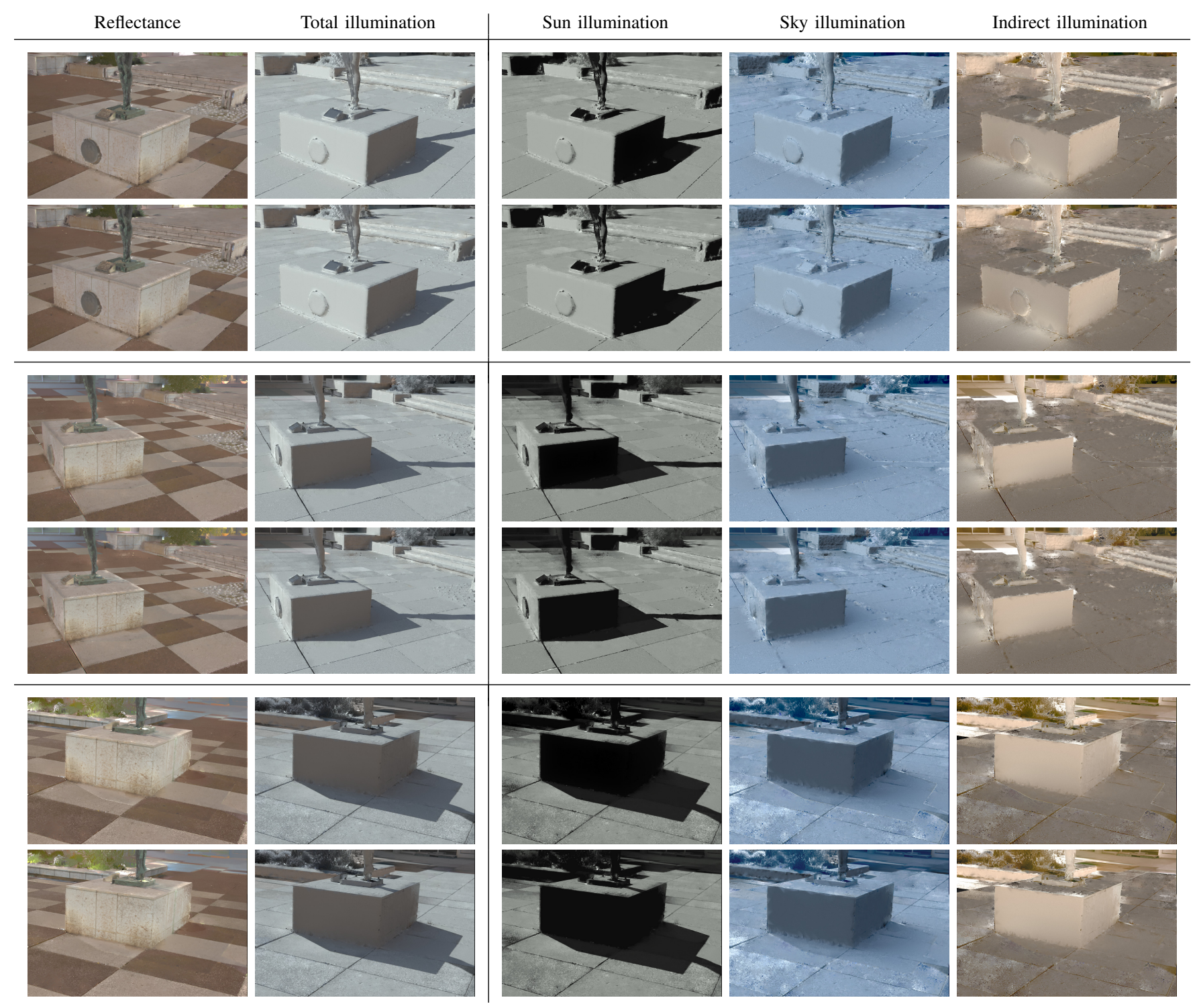

TABLE 4: Results of our decomposition on the Statue scene before (top) and after final inpainting (bottom). The linear images have been scaled for display, before applying gamma correction with $\gamma=1 / 2.2$; the scaling values were: 0.6 for the input, 1 for the reflectance, 0.15 for total illumination and sun illumination, 0.6 for sky illumination, 1 for indirect illumination. 


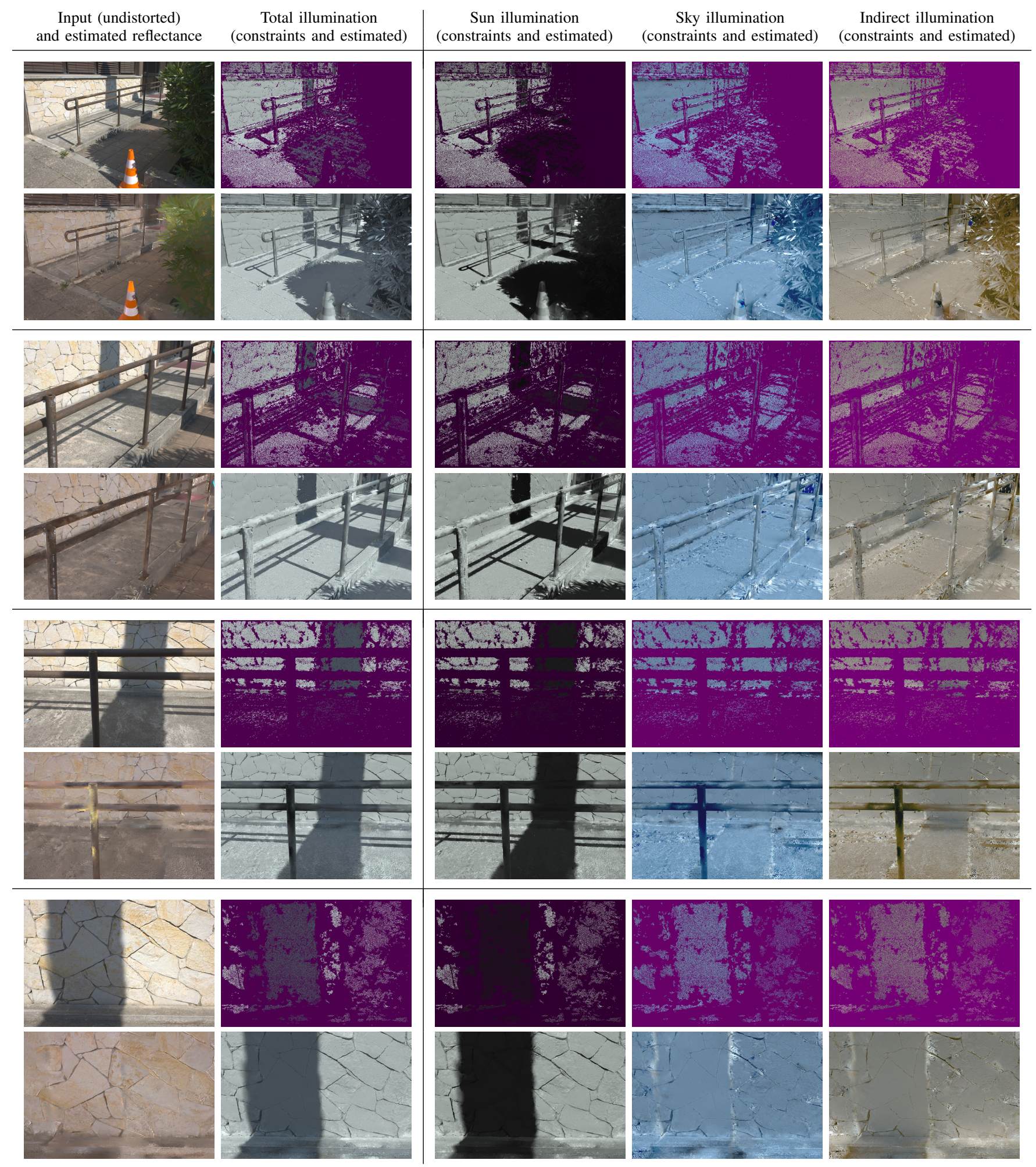

TABLE 5: Results of our decomposition on the Ramp scene after final inpainting. The linear images have been scaled for display, before applying gamma correction with $\gamma=1 / 2.2$; the scaling values were: 0.4 for the input, 1 for the reflectance, 0.08 for total illumination and sun illumination, 0.45 for sky illumination, 0.6 for indirect illumination. 

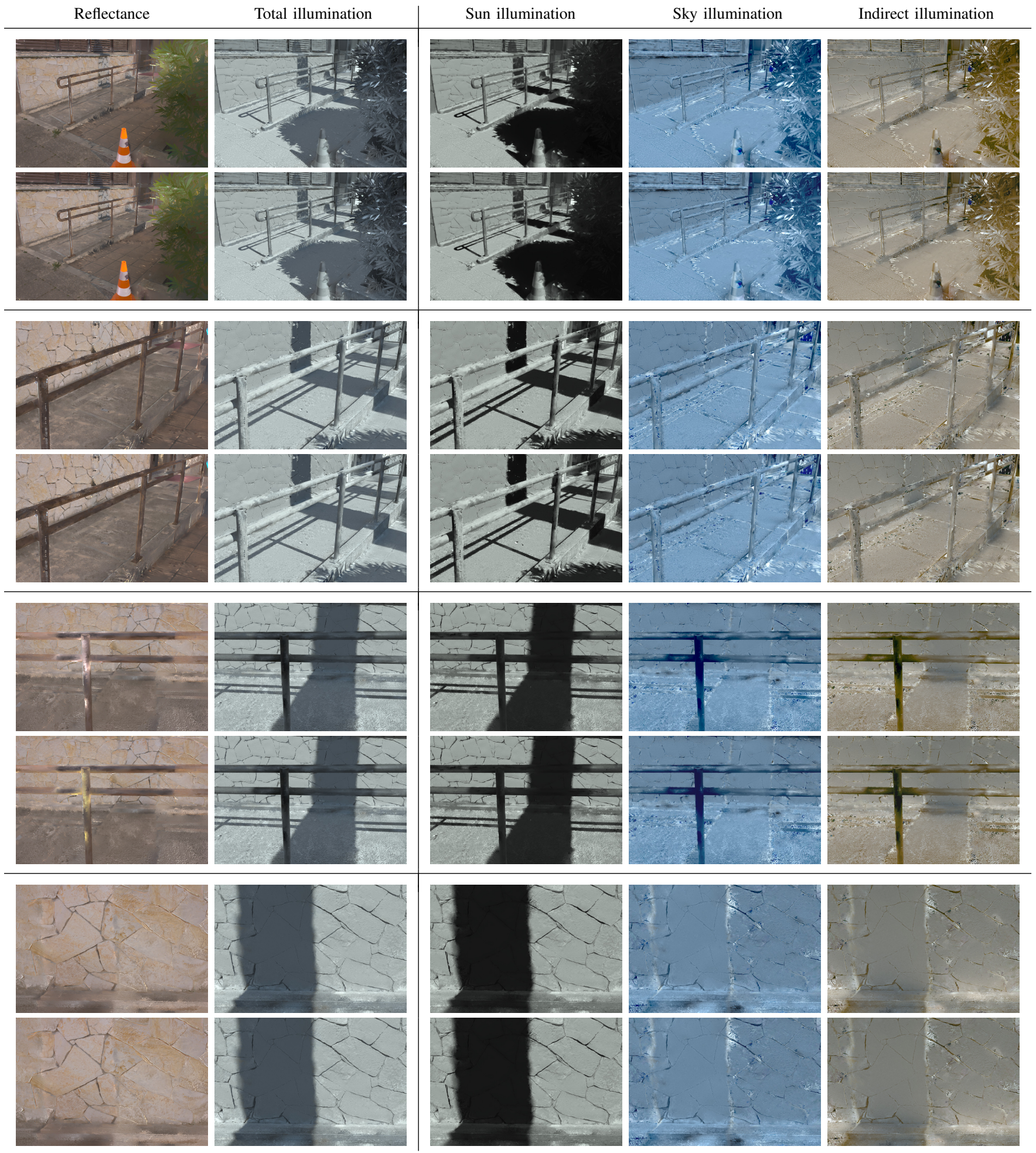

TABLE 6: Results of our decomposition on the Ramp scene before (top) and after final inpainting (bottom). The linear images have been scaled for display, before applying gamma correction with $\gamma=1 / 2.2$; the scaling values were: 0.4 for the input, 1 for the reflectance, 0.08 for total illumination and sun illumination, 0.45 for sky illumination, 0.6 for indirect illumination. 
Input (undistorted) and estimated reflectance
Total illumination (constraints and estimated)
Sun illumination (constraints and estimated)
Sky illumination

(constraints and estimated)
Indirect illumination (constraints and estimated)
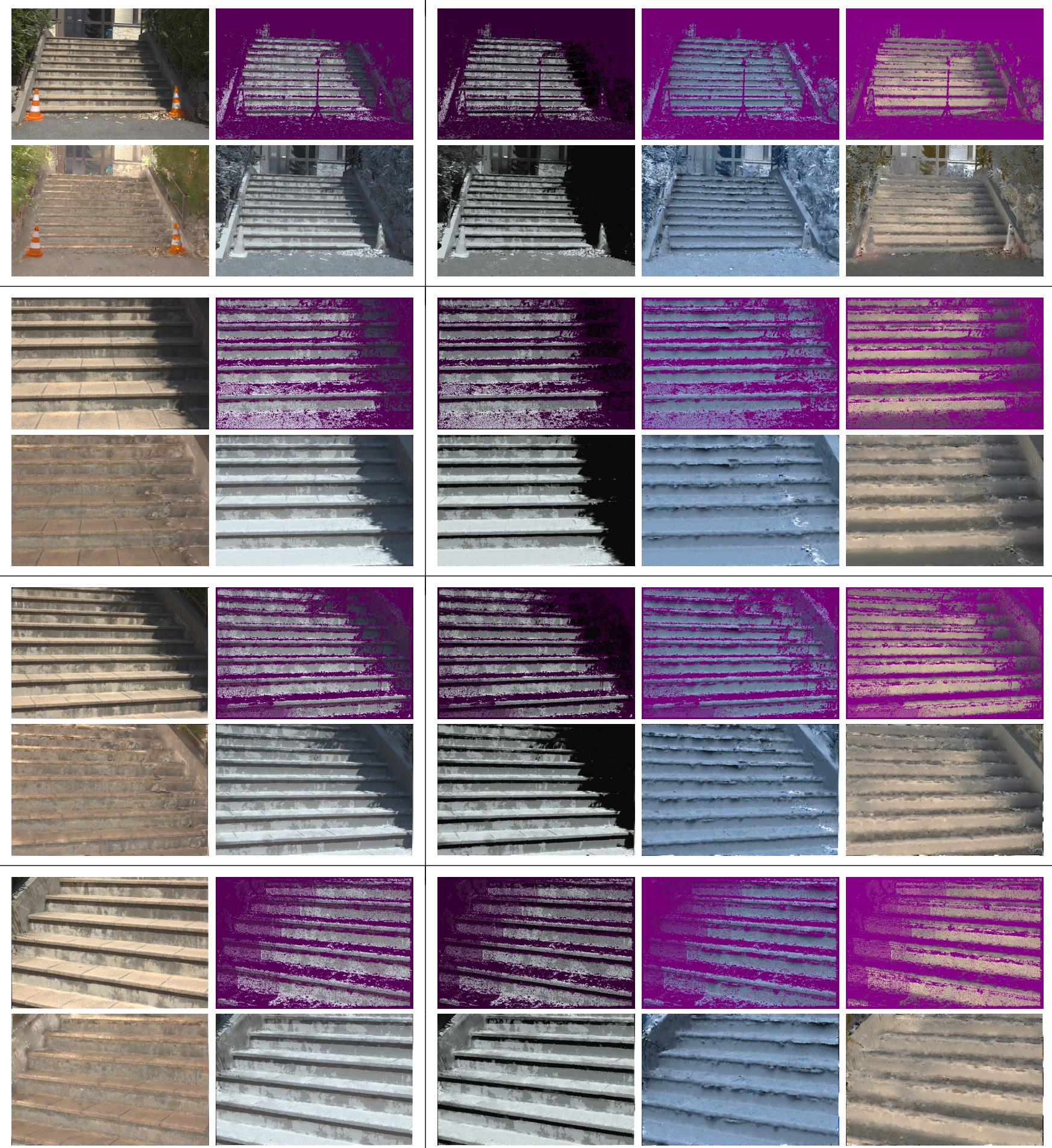

TABLE 7: Results of our decomposition on the Stairs scene after final inpainting. The linear images have been scaled for display, before applying gamma correction with $\gamma=1 / 2.2$; the scaling values were: 0.5 for the input, 1.5 for the reflectance, 0.1 for total illumination and sun illumination, 0.6 for sky illumination, 0.8 for indirect illumination. 


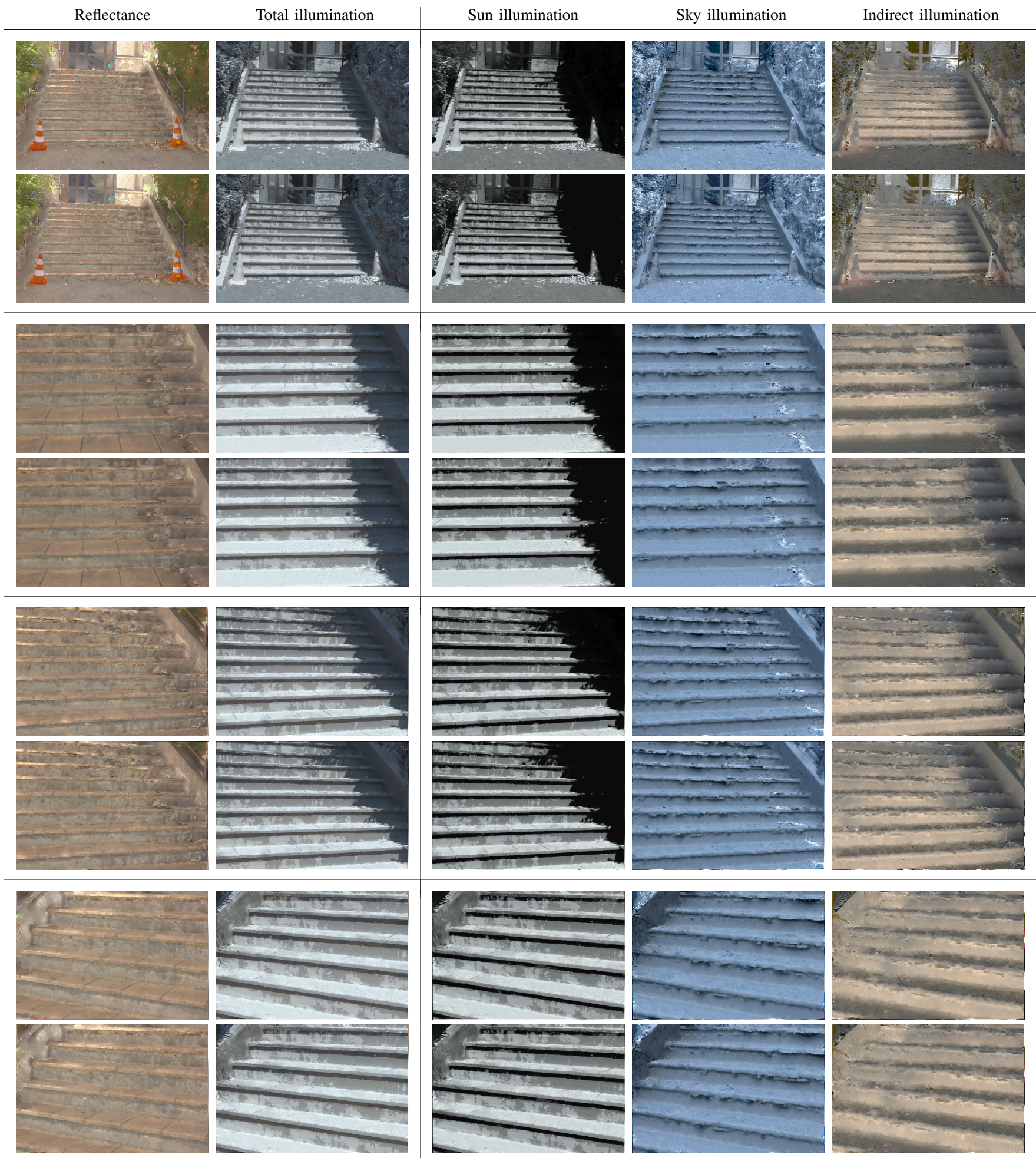

TABLE 8: Results of our decomposition on the Stairs scene before (top) and after final inpainting (bottom). The linear images have been scaled for display, before applying gamma correction with $\gamma=1 / 2.2$; the scaling values were: 0.5 for the input, 1.5 for the reflectance, 0.1 for total illumination and sun illumination, 0.6 for sky illumination, 0.8 for indirect illumination. 